\title{
Emerging Trends in T-cell based Vaccines
}

\author{
Shikha Tarang ${ }^{1 *}$ and Varun Kesherwani ${ }^{2}$ \\ ${ }^{1}$ Department of Oral Biology, Creighton University, USA \\ ${ }^{2}$ Child Research Institute, University of Nebraska Medical Center, USA \\ *Corresponding author: Shikha Tarang, Department of Oral Biology, Creighton University, Omaha-NE68178, USA. \\ To Cite This Article: Shikha Tarang, Emerging Trends in T-cell based Vaccines. 2020 - 7(5). AJBSR.MS.ID.001196. \\ DOI: 10.34297/AJBSR.2020.07.001196.
}

Received: 眥 February 14, 2020; Published: 制 March 02, 2020

\section{Opinion}

Since Edward Jenner's first use of cowpox pustules against smallpox to the present use of subunit vaccines, vaccinology has seen a paradigm shift. The transition from time-intensive microbiological approaches to the current machine learningbased prediction algorithms for epitope identification have enabled the development of faster, cost-effective and efficient vaccines. However, to outrace the newer and deadlier emerging pathogens, our approach to vaccination needs to be constantly modified. As we mark the final year of the Decade of Vaccines and its product Global Vaccine Action Plan (GVAP), we emphasize the significance of T- cell responses to achieve a successful vaccination.

While the traditional vaccination approaches mostly relied on antibody generation as a measure of vaccine efficacy; recent years have seen a rekindled interest in T-cells' role for immunoprotection. In fact, current vaccination approaches against a number of pathogens are exclusively designed to elicit T- cell-based immune response. Such an approach is particularly useful in persistent latestage chronic viral infections, where the body is unable to clear the infection (such as human immunodeficiency virus (HIV) [1], hepatitis C virus (HCV) [2], cytomegalovirus (CMV) [3], vaccinia virus [4]). Moreover, T-cell responses show a greater magnitude, affinity, and multifunctionality which can be tailored against the invading pathogens. In addition to its obvious advantage in chronic viral infections, T- cell-based immunity is widely used against intracellular bacteria [5] and is also currently being targeted in cancer [6], autoimmune disorders [7], allergies [8], and even fibrosis [9].

The success of any vaccination depends on the identification of effective epitopes, which is greatly expedited by the use of computational tools (referred to as immunoinformatics). For $\mathrm{T}$ - cell epitope mapping, two distinct approaches exist. In an epitope-based approach, top- most antigenic epitopes are selected by screening a subset of proteins or the entire proteome of an organism. Such an approach eliminates non-immunogenic sequences and is therefore expected to elicit a robust immune response [10]. Overlapping peptides uses a library of promiscuous peptides to stimulate T- cell responses without knowledge of the epitopes or individual's genetic makeup [11]. In contrast to antibody response, antigen recognition by both CD4+ and CD8+ T- cells require their presentation in complex with major histocompatibility complex (MHC) displayed on the surface the antigen-presenting cell (APC) (CD4+ T- cells are MHC class II-restricted; while CD8+ T- cells are MHC class I restricted). The binding groove of MHC's is formed by a single $\alpha$-chain (MHCI), an $\alpha$-chain and a $\beta$ - chain (MHCII) and interacts with sidechains of the antigenic peptides' amino acid residues. MHC, and analogous human leukocyte antigen (HLA), are the most polymorphic loci in the human genome.Each MHC class consists of three genetic loci (MHCI - HLA-A, -B, -C; MHCII - HLA-DR, -DP, -DQ) [12]. Thus, an individual's genetic makeup plays a major role in determining the nature of the peptide-MHC complex recognized by T- cells. The structural binding between antigenic peptides and MHC molecules can be also predicted in silico using computer algorithms and modeling. This offers a unique opportunity for the development of personalized vaccines based on an individual's HLA allele frequency. Personalized vaccines potentiate the immune response to the administered vaccine by eliminating non-reactive responses and are currently being developed for HCV [13], measles [14] and several cancers [15]. Moreover, understanding of the association between an individual's genetic makeup and immune response to the administered vaccines is important to predict the success of immunization where HLA polymorphism is known to confer a variability in immune response (E.g., the adverse reaction in case of vaccinia virus [16]). Such an approach can also be adopted for immunocompromised individuals, attenuating the risk of an 
adverse event. Recently, we have identified peptides against all 12 superfamilies of HLAI and 27 reference alleles of HLAII, which can be used in developing HLA specific personalized vaccine in BK viremia [17]. Thus, the beauty of T-cell based vaccine is that while on one hand it can be designed to achieve a personalized response (using specific HLA epitopes), it can be also be developed as a universal vaccine (using epitopes that can bind to all major HLA molecules).

The advancement in epitope identification is (unfortunately) not met with equivalent technical advancements in experimental validation of the identified targets.The common assays for immunogenicity testing include T-cell proliferation and cytokinebased assays (ELISPOT, ICS, ELISA) in peripheral blood mononuclear cells (PBMCs). Flow cytometer or CyTOF technology-based T-cell signature cytokine profiling (IFN- $\gamma$, IL-2, IL-17, TNF- $\alpha$, etc.) is also done to assess a functional immune response. Though PBMC may not faithfully mirror immune response in primary lymphoid organs, nonetheless, their accessibility (particularly in humans) and ease of culture makes them the most popular choice for immunogenicity testing [18]. NGS-based deep sequencing of TCR receptors directly measures T- cell diversity upon vaccination [19]. Before proceeding to the clinical trials, studies in animal models provide information on vaccine safety, efficacy, optimal dose, formulation and route of delivery. While mice are the most common species for vaccine research, the similarity in disease pathogenesis dictates rabbits as the primary experimental models a number of human infections [20]. Overall, animals show a higher immune response than humans (which also show a variable response due to genetic polymorphism). Peptides are delivered either as a DNA vaccine in viral vectors (most commonly adenovirus) or as a vaccine cocktail [21,22]. To enhance their immunogenicity epitope-based vaccines also require the use of adjuvants. There is not much research on T-cellspecific adjuvant development. The most common adjuvants are pathogen-associated molecular pattern (PAMP) based, which can be added in the vaccine cocktail or molecularly linked to the vaccine constructs [23]. Viral vectors also improve the immunogenicity of the immunodominant epitopes in vivo [21]. Another challenge to T-cell-based vaccination is the lack of long-term memory T-cell formation with antigens requiring repeated vaccination [21,24]. Future research in this direction also requires the development of adjuvants that can generate specific Th responses (Th1or Th17). Targeting the T-cell inhibitory molecules like PD-1 and CTLA-4 or T-cell metabolism can maximize T-cell activation and are currently being utilized [24].

Vaccines are the single-most-important factor in eradicating a number of infectious diseases and greatly reducing the prevalence of many more. We have come a long way since the empirical approach to vaccination, to a much better understanding of how the specific vaccine components activate the immune system. However, despite significant technological and biological advancements, the generation of vaccine-based immunoprotection still remains a challenge for many existing diseases. Moreover, newly emerging deadly pathogens of epidemic potential-such as Zika, Ebola, Coronavirus - will continue to fuel our constant war against microbial pathogens.

\section{References}

1. Hansen SG, Ford JC, Lewis MS (2011) Profound early control of highly pathogenic SIV by an effector memory T-cell vaccine. Nature 473(7348): 523-527.

2. Swadling L, Capone S, Antrobus RD (2014) A human vaccine strategy based on chimpanzee adenoviral and MVA vectors that primes, boosts, and sustains functional HCV-specific T cell memory. Sci Transl Med 6(261): 261ra153.

3. Panagioti E, Redeker A, van Duikeren S (2016) The breadth of synthetic long peptide vaccine induced CD8+ $\mathrm{T}$ cell responses determines the efficacy against mouse cytomegalovirus infection. PLoS Pathog 12(9): e1005895.

4. Calvo Calle JM, Strug I, Nastke MD, Baker SP, Stern LJ, et al. (2007) Human CD4+ T cell epitopes from vaccinia virus induced by vaccination or infection. PLoS Pathog 3(10): 1511-1529.

5. Thakur A, Mikkelsen H, Jungersen G (2019) Intracellular pathogens: Host immunity and microbial persistence strategies. J Immunol Res 2019: 1356540 .

6. Kartikasari AER, Prakash MD, Cox M (2019) Therapeutic cancer vaccines-T cell responses and epigenetic modulation. Front Immunol 9: 3109.

7. Skapenko A, Leipe J, Lipsky PE, Schulze-Koops H (2005) The role of the T cell in autoimmune inflammation. Arthritis Res Ther 7 (Suppl 2): S4-S14.

8. Woodfolk JA (2007) T-cell responses to allergens. J Allergy Clin Immunol 119(2): 280-296.

9. Aghajanian H, Kimura T, Rurik JG (2019) Targeting cardiac fibrosis with engineered T cells. Nature 573(7774): 430-433.

10. Sette A, Fikes J (2003) Epitope-based vaccines: An update on epitope identification, vaccine design and delivery. Curr Opin Immunol 15(4): 461-470.

11. Jiang S, Song R, Popov S, Mirshahidi S, Ruprecht RM (2006) Overlapping synthetic peptides as vaccines. Vaccine 24(37-39): 6356-6365.

12. Wieczorek M, Abualrous ET, Sticht J (2017) Major histocompatibility complex (MHC) class I and MHC class II proteins: Conformational plasticity in antigen presentation. Front Immunol 8: 292.

13. Yutani S, Yamada A, Yoshida K (2007) Phase I clinical study of a personalized peptide vaccination for patients infected with hepatitis $\mathrm{C}$ virus (HCV) $1 \mathrm{~b}$ who failed to respond to interferon-based therapy. Vaccine 25(42): 7429-7435.

14. Poland GA, Kennedy RB, Ovsyannikova IG (2011) Vaccinomics and personalized vaccinology: Is science leading us toward a new path of directed vaccine development and discovery? PLoS Pathog 7(12): e1002344.

15. Sahin U, Tureci O (2018) Personalized vaccines for cancer immunotherapy. Science 359(6382): 1355-1360.

16. Poland GA, Ovsyannikova IG, Jacobson RM (2008) Personalized vaccines: The emerging field of vaccinomics. Expert Opin Biol Ther 8(11): 16591667.

17. Kesherwani V, Tarang S (2019) An immunoinformatic approach to universal therapeutic vaccine design against BK virus. Vaccine 37(26): 3457-3463. 
18. Saade F, Gorski SA, Petrovsky N (2012) Pushing the frontiers of T-cell vaccines: Accurate measurement of human T-cell responses. Expert Rev Vaccines 11(12): 1459-1470.

19. Pogorelyy MV, Minervina AA, Touzel MP (2018) Precise tracking of vaccine-responding $\mathrm{T}$ cell clones reveals convergent and personalized response in identical twins. Proc Natl Acad Sci U S A 115(50): 1270412709.

20. Peng X, Knouse JA, Hernon KM (2015) Rabbit models for studying human infectious diseases. Comp Med 65(6): 499-507.

21. Robinson HL, Amara RR (2005) T cell vaccines for microbial infections. Nat Med 11(4 Suppl): S25-32.
22. Miyazawa M, Katsuda M, Maguchi H (2017) Phase II clinical trial using novel peptide cocktail vaccine as a postoperative adjuvant treatment for surgically resected pancreatic cancer patients. Int J Cancer 140(4): 973982.

23. Hayashi T, Momota M, Kuroda E (2018) DAMP-inducing adjuvant and PAMP adjuvants parallelly enhance protective type- 2 and type- 1 immune responses to influenza split vaccination. Front Immunol 9: 2619.

24. Panagioti E, Klenerman P, Lee LN, van der Burg SH, Arens R, et al. (2018) Features of effective $\mathrm{T}$ cell-inducing vaccines against chronic viral infections. Front Immunol 9: 276. 